\title{
Prevalence of Chronic Periodontitis: A Retrospective Cross-sectional Study
}

\author{
Ilma Robo $^{1 *}$, Saimir Heta ${ }^{2}$, Geriona Lasku ${ }^{3}$, Vera Ostreni ${ }^{1}$ \\ ${ }^{1}$ Department of Periodontology, Faculty of Medical Sciences, Albanian University, Tirana, Albania. \\ ${ }^{2}$ Pediatric Surgery, University Hospital Center of Tirana, RR. Dibra, 370, Tirana, Albania. \\ ${ }^{3}$ Private Dental Clinic, Tirana, Albania.
}

Received 04 December 2020; Revised 15 February 2021; Accepted 26 February 2021; Published 01 March 2021

\begin{abstract}
Introduction: The characteristic appearance of chronic periodontitis with the absence of bacterial plaque or with minimal amounts of it, but with the presence of gingival health, expressed in the specific pink color of the gingiva, is associated with active stages of the disease and its inactive stages. The aim of this study is to compare cross-sectional and retrograde data on the prevalence of chronic periodontitis in the two respective groups of patients, evaluated according to specific periodontal diagnostic tools. Materials and Methods: The study was applied in two groups of patients: the first group of patients presented at the dental clinic in the period October 2019 - January 2020 and the second group; in the period October-November in the annual interval 2010-2018. In these patients, periodontal status was assessed based on probing values, bacterial plaque index, bleeding index, and amount of gingival fluid in specific dentures. The number of natural teeth and the number of teeth replaced with fixed prosthetics were recorded. Results: For both cross-sectional and retrograde data, chronic periodontitis is a disease that affects young people, with a higher prevalence in females. This data is expressed with high ratios of tooth loss for periodontal reasons. The highest dental care for replacement with fixed prosthetics, again belongs to the female sex, but with a high bacterial plaque index. The high index of gingival hemorrhage with reduced bacterial plaque index, are data with high percentages both for the group of patients crosssectional data and for the group of patients with retrograde data. Conclusions: The high prevalence of chronic periodontitis at a young age indicates a poor prognosis of this disease at older ages. Gingival recession associated with loss of attachment for patients with chronic periodontitis have higher values at age intervals 31-50 years, intervals where systemic pathologies are in the stages of development and stabilization in the human body.
\end{abstract}

Keywords: Chronic Periodontitis; Cross-Sectional; Retrograde; Prevalence.

\section{Introduction}

Patients with chronic periodontitis are infected with Actinomyces Actinomycetemcomitans [1-4]. It is this bacterium that, under the conditions of the patient's marked decline in immunity, attacks the tooth-bearing structures, orienting the bone loss mainly towards the horizontal bone loss. The prevalence of chronic periodontitis is high and there is no age preference. Generally, all ages are affected, this in combination with the condition of infection at the right time by the specific bacterium with good oral hygiene; conditions that must simultaneously be in combination with each other. The study aims to collect retrospective and cross-sectional data on the prevalence of chronic

* Corresponding author: ilmarobo@yahoo.com

dol http://dx.doi.org/10.28991/SciMedJ-2021-0301-5

> This is an open access article under the CC-BY license (https://creativecommons.org/licenses/by/4.0/).

(C) Authors retain all copyrights. 
periodontitis [5-10]. Having both types of studies for the purpose of data collection, it is possible to find possible correlations on the effect of risk factors for the occurrence of chronic periodontitis, the effect of local dental factors on the formation of bacterial plaque and then on the occurrence of chronic, local or generalized periodontitis, or the extent of this infection divided or not depending on the sex, age of the affected individuals. An important element that gives the opportunity through this study to collect and process the recorded data, is the possibility of finding correlations of this diagnosis and the presence of other lesions of hard and soft tissues [2, 5, 7, 11-15].

Chronic periodontitis passes into active and inactive stages, during which the patient complains of itching sensation, the sensation that increases, so much so that it forces the patient to exert more force in the usual brushing of teeth. Decreased immunity can be a flu condition, the first day of sun exposure, the first day of menstruation, reasons which lead to subsequent decline in immunity. There are cases when the transition from the inactive phase to the active phase may not be felt by the patient. What happens to the dental structures and mainly to the periodontal ligament, is its resorption, or the fusion of collagenous fibers with the tendency for horizontal bone loss [3, 16-20].

Even the patient's tendency to brush his teeth with a higher than normal force pushes even further the tendency for horizontal loss in the affected areas. The gingiva follows the bone level, supporting exactly the clinical picture stated above. So, the gingiva is pink, due to good oral hygiene, but the interdental papilla does not completely fill the space from the point of contact to the beginning of the gingival sulcus, so there are empty spaces below the point of contact, but all are physiological and functional normal [21, 22].

\section{Materials and Methods}

Comparing the figures recorded and conceived at two different times and moments requires the analysis of crosssectional and retrospective data on the prevalence of chronic periodontitis. In itself, the purpose of this study involves recording data on the prevalence of chronic periodontitis at as diverse and varied age intervals as possible. In this study, data were collected on two groups of patients with the aim of fulfilling the purpose of the study. The first group includes patients who presented ad-hoc in the period October 2019 - January 2020, at the Dental Clinic of Albanian University, for routine periodontal treatment.

The second group of patients are patients presented in the period October-November in the time interval 20102018, patients of the Dental Clinic of Albanian University. These are patients presented each year in the monthly period mentioned above. Agreeing to be included in the study verbally was a condition of the patients involved in the study, this for the patients of the first group. Of course, the agreement to be included in the study has the principle of preserving the anonymity of the recorded data. For the patients of the second group, the data recording was performed in the completed periodontal cards at the respective times. Each periodontal card is initialed by the patient.

At patients of both groups, demographic data were recorded, the number of natural teeth in the oral cavity and the number of teeth replaced with fixed prosthetic works. In this study for these patients, the following data were recorded: gingival hemorrhage index, bacterial plaque index and the amount of gingival fluid in the mandibular canine and maxillary molar. Near the dental chair, the ways of diagnosing xerostomia are different. In our study a simple way was selected. Using filter paper, which rests on the dorsal surface of the tongue for 5 seconds, wet surface was recorded during this time period. If the surface of the filter paper is $1 \mathrm{~cm}$ or more cannot be spoken about the presence of xerostomia, if this area is less than $1 \mathrm{~cm}$, then it is accepted the presence of xerostomia. The presence of candidiasis is examined by visual examination and then by palpation. Palpation examination is performed to see if the white candidiasis lesion is scaly or not from the mucosal surface.

The white lesion is candidiasis everywhere on the surface of the mucous membrane and the red lesion on the surface of the fixed mucosa. Symptoms of itching and burning of the surface are in both cases. Red absorbent paper was used to measure gingival fluid. Red absorbent paper or paper-point, used to dry root canals during endodontic treatments, is the most common means of measuring gingival fluid. Duration is 1 minute, positioned inside the gingival sulcus. Then, after the red endodontic paper-point is removed from the gingival sulcus, measure how many millimeters it is wet. The amount of gingival fluid produced in one of the areas on the tooth surface, based on literature data, on red paper-point absorbent paper placed for 1 minute inside the sulcus, wet $3 \mathrm{~mm}$ is normal. The highest amount, i.e. higher than $3 \mathrm{~mm}$, and the reduced amount is in cases when the absorbent paper is wet less than $3 \mathrm{~mm}$.

\section{Results}

After collecting the data and registering the excel spreadsheet, the following statistical processing was performed. Patients involved in the collection and processing of cross-sectional study data were termed as first-group patients and patients for retrograde data were termed as second-group patients. 
Table 1. Data on the gender of patients included in the study: The first group and the second group

\begin{tabular}{cccccccc}
\hline & Cross-secional group & $\%$ & $\begin{array}{c}\text { Retrograde } \\
\text { group }\end{array}$ & $\%$ & Total & $\%$ & $\begin{array}{c}\text { Cumulative } \\
\text { percentage }\end{array}$ \\
\hline Female & 23 & $23 \%$ & 26 & $27 \%$ & 49 & $50 \%$ & 50 \\
Male & 11 & $11 \%$ & 38 & $39 \%$ & 49 & $50 \%$ & $100 \%$ \\
Total & 34 & $35 \%$ & 64 & $65 \%$ & 98 & $100 \%$ & \\
\hline
\end{tabular}

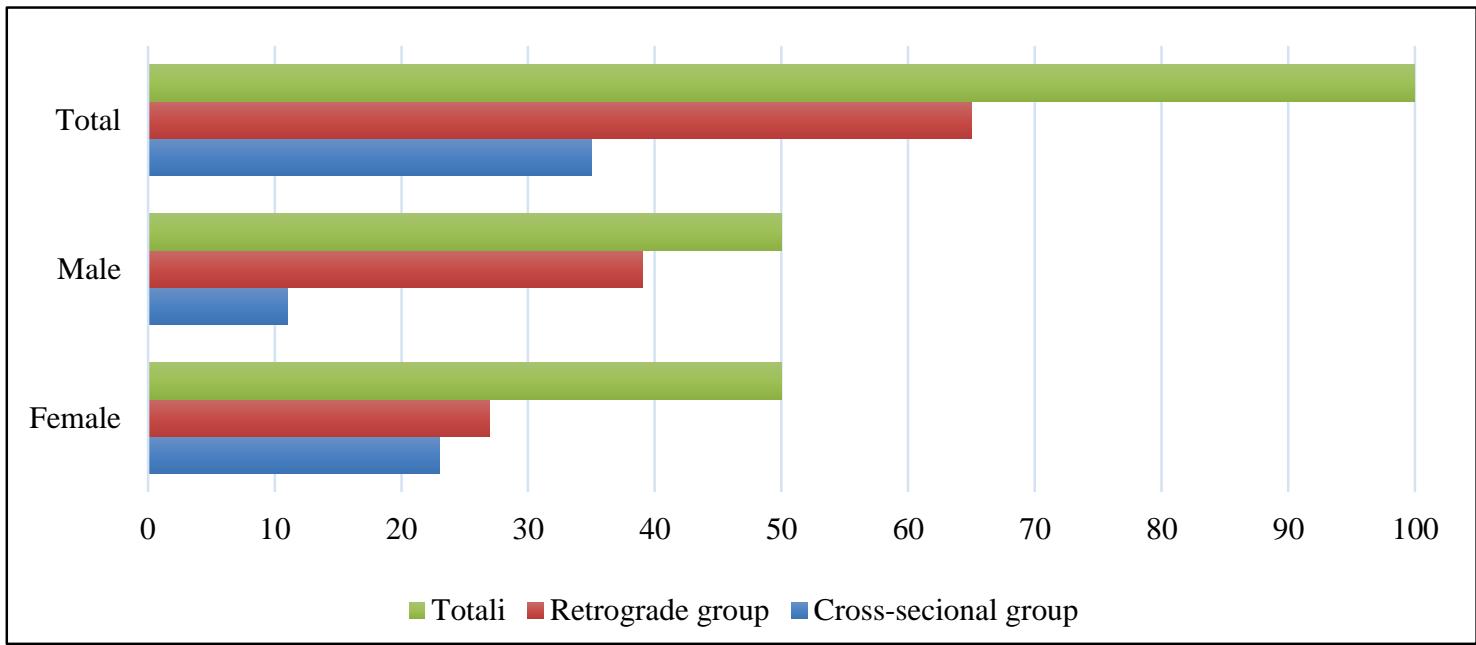

Figure 1. This graph presents data on the gender of patients included in the study: the first group and the second group

This table presents the male-female demographic data, according to the age division for the first group and the second group.

Table 2. Gender-age, first group and second group patients

\begin{tabular}{cccccc}
\hline & Male & $\boldsymbol{\%}$ & Female & $\%$ & Cumulative age percentage \\
\hline Age 20-30 years & 29 & $30 \%$ & 24 & $24 \%$ & $56 \%$ \\
Age 31-50 years & 11 & $11 \%$ & 13 & $13 \%$ & $80 \%$ \\
Age 51-years & 9 & $9 \%$ & 12 & $12 \%$ & $100 \%$ \\
Total & 49 & $50 \%$ & 49 & $50 \%$ & \\
\hline Cumulative percentage gender & & $\mathbf{5 0 \%}$ & & $\mathbf{1 0 0 \%}$ & \\
\hline
\end{tabular}

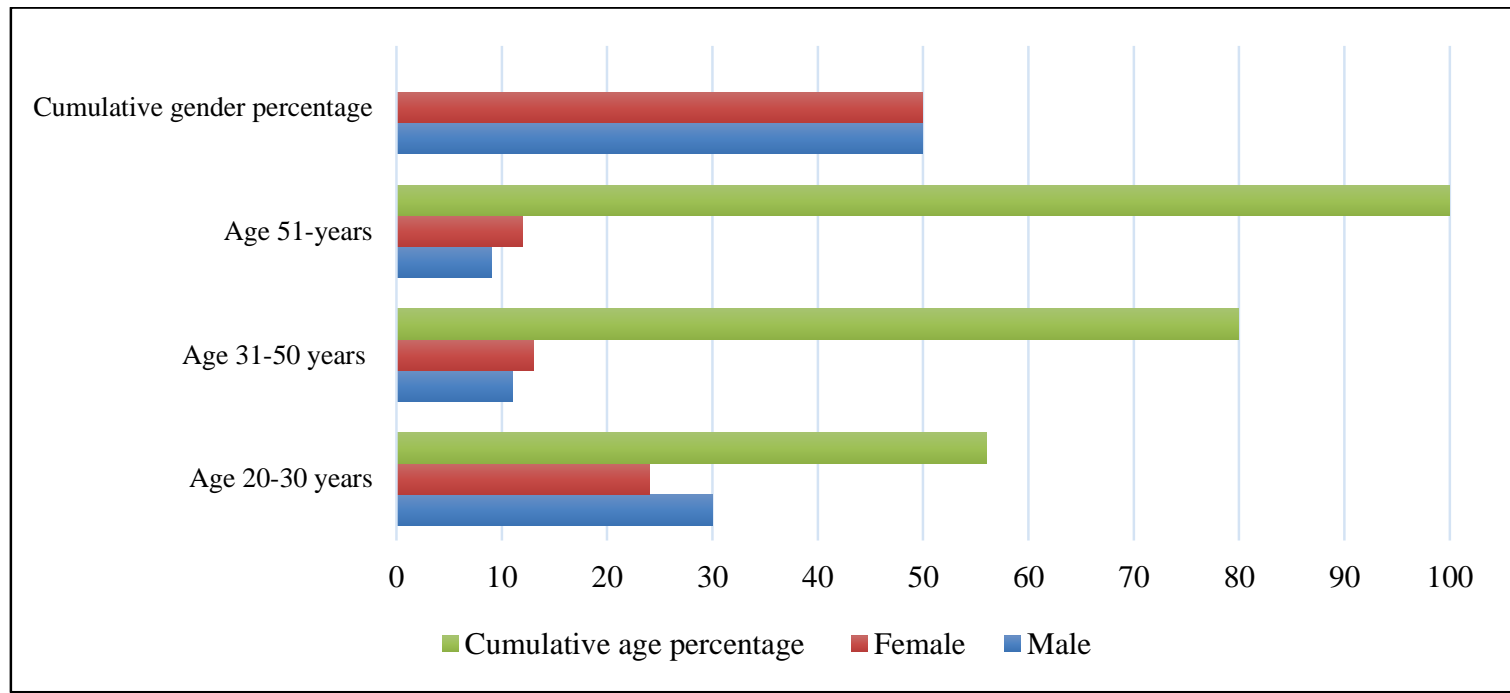

Figure 2. This graph presents the male-female demographic data, according to the age division for the first group and the second group

Table 3 shows the recorded data on dental health status, for the group of patients on cross-sectional and retrograde data. 
Table 3. Natural tooth number-gender data, for the first and second group of patients

\begin{tabular}{ccccccc}
\hline Natyral teeth & Female & $\%$ & Male & $\%$ & Total & $\%$ \\
\hline Number till 10 & 9 & $9 \%$ & 9 & $9 \%$ & 18 & $18 \%$ \\
Number 11-20 & 12 & $12 \%$ & 10 & $10 \%$ & 22 & $22 \%$ \\
Number 20-31 & 17 & $28 \%$ & 23 & $23 \%$ & 40 & $52 \%$ \\
Number 32 & 11 & $11 \%$ & 7 & $8 \%$ & 18 & $19 \%$ \\
\hline Total & 49 & $50 \%$ & 49 & $50 \%$ & 98 & $100 \%$ \\
\hline
\end{tabular}

Table 4 shows the recorded data on dental health status, for the group of patients on cross-sectional and retrograde data.

Table 4. Gender-replaced tooth number data, for the first and second group of patients

\begin{tabular}{ccccccc}
\hline Natyral teeth & Female & $\%$ & Male & $\%$ & Total & $\%$ \\
\hline Number till 10 & 12 & $12 \%$ & 9 & $10 \%$ & 21 & $12 \%$ \\
Number 11-20 & 4 & $4 \%$ & 3 & $3 \%$ & 7 & $7 \%$ \\
Number 20-31 & 2 & $2 \%$ & 1 & $1 \%$ & 3 & $3 \%$ \\
Number 32 & 4 & $4 \%$ & 3 & $3 \%$ & 7 & $7 \%$ \\
\hline Total & 22 & $22 \%$ & 16 & $17 \%$ & 38 & $29 \%$ \\
\hline
\end{tabular}

The Table 5 present data regarding plaque index and bleeding index depending on the patient's gender.

Table 5. Gender-healthy patient data, based on periodontal indices, for the first group of patients

\begin{tabular}{ccccccc}
\hline Healthy Patients & Female & $\%$ & Male & $\%$ & Total & $\%$ \\
\hline Indexes & 10 & $29 \%$ & 3 & $9 \%$ & 13 & $38 \%$ \\
Index of pllaque & 5 & $15 \%$ & 1 & $3 \%$ & 6 & $18 \%$ \\
Index of pllaque/hemorraghie & 3 & $9 \%$ & 0 & $0 \%$ & 3 & $9 \%$ \\
\hline
\end{tabular}

Table 6 shows the recorded data on dental health status, for the group of patients on retrograde data.

Table 6. Data on healthy patients - gender, based on periodontal indices, for the second group of patients

\begin{tabular}{ccccccc}
\hline Healthy Patients & Female & $\%$ & Male & $\%$ & Total & $\%$ \\
\hline Indexes & 15 & $23 \%$ & 7 & $11 \%$ & 22 & $34 \%$ \\
Index of pllaque & 12 & $19 \%$ & 5 & $9 \%$ & 17 & $28 \%$ \\
Index of hemorraghie & 8 & $13 \%$ & 2 & $3 \%$ & 10 & $16 \%$ \\
\hline Index of pllaque/hemorraghie & 8 &
\end{tabular}

The assessment of pathology also depends on the aggravation of the clinical situation. In this logic in Tables 7 and 8 are reflected the fluctuations of the amount of gingival fluid produced, measured in wet millimeters of paper point.

Table 7. The amount of gingival fluid shown in aggravation levels of the clinical situation, patients first group

\begin{tabular}{|c|c|c|c|c|}
\hline Gingival fluid Patients & Mandibular Canine & $\%$ & Maxillary Molar & $\%$ \\
\hline $0-3 \mathrm{~mm}$ & 16 & $47 \%$ & 13 & $38 \%$ \\
\hline $4-6 \mathrm{~mm}$ & 8 & $24 \%$ & 8 & $24 \%$ \\
\hline $7-10 \mathrm{~mm}$ & 10 & $29 \%$ & 13 & $38 \%$ \\
\hline Total & 34 & $100 \%$ & 34 & $100 \%$ \\
\hline
\end{tabular}


Table 8. Amount of gingival fluid shown in aggravation levels of clinical situation, patients second group

\begin{tabular}{|c|c|c|c|c|}
\hline Gingival fluid Patients & Mandibular Canine & $\%$ & Maxillary Molar & $\%$ \\
\hline $0-3 \mathrm{~mm}$ & 34 & $53 \%$ & 26 & $41 \%$ \\
\hline $4-6 \mathrm{~mm}$ & 17 & $27 \%$ & 23 & $36 \%$ \\
\hline $7-10 \mathrm{~mm}$ & 13 & $20 \%$ & 15 & $23 \%$ \\
\hline Total & 64 & $100 \%$ & 64 & $100 \%$ \\
\hline
\end{tabular}

Figure 3 and figure 4 show the division into norm and pathology, the latter mild or aggravated in all patients, respectively for the mandibular canine and the maxillary molar.

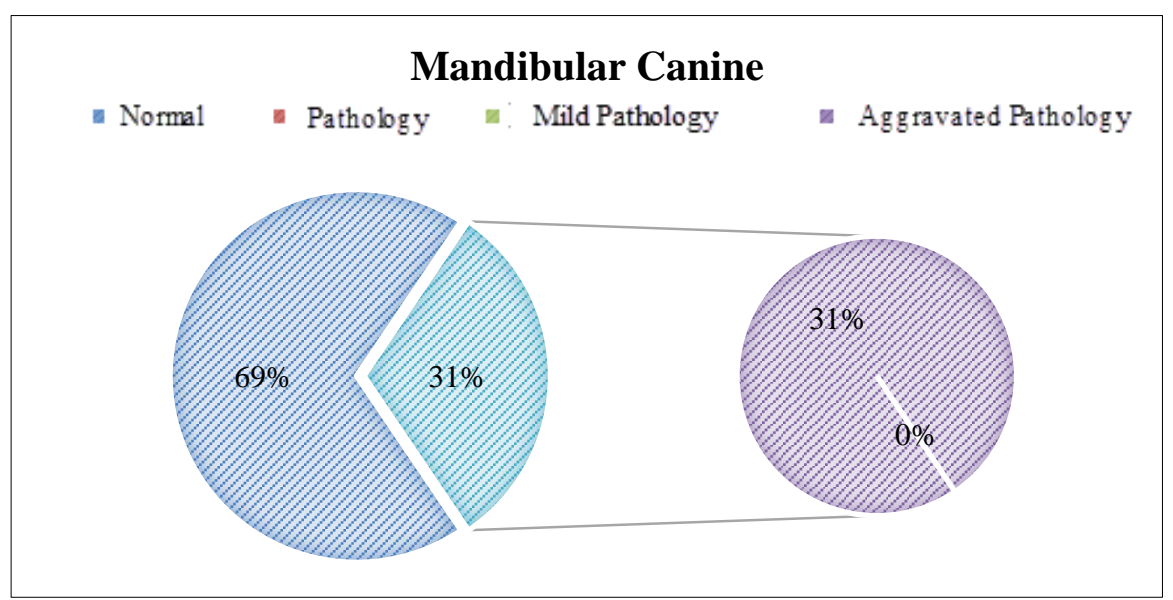

Figure 3. This graph presents the recorded data on the amount of gingival fluid, measured in the mandibular canine

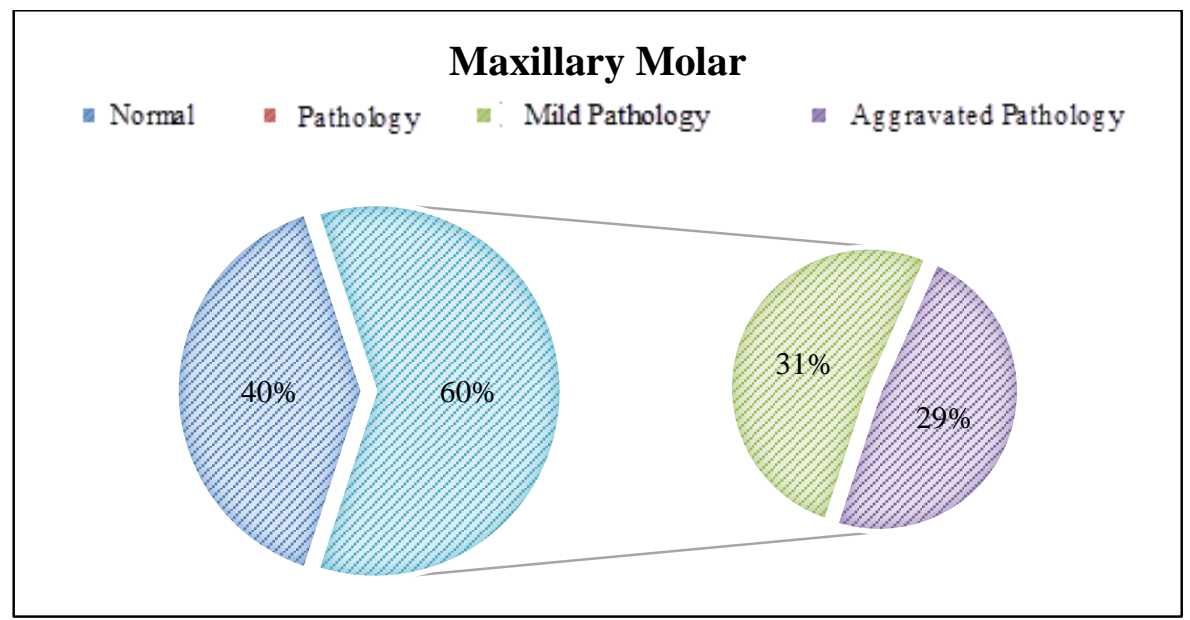

Figure 4. This graph presents the recorded data on the amount of gingival fluid, measured at the maxillary molar
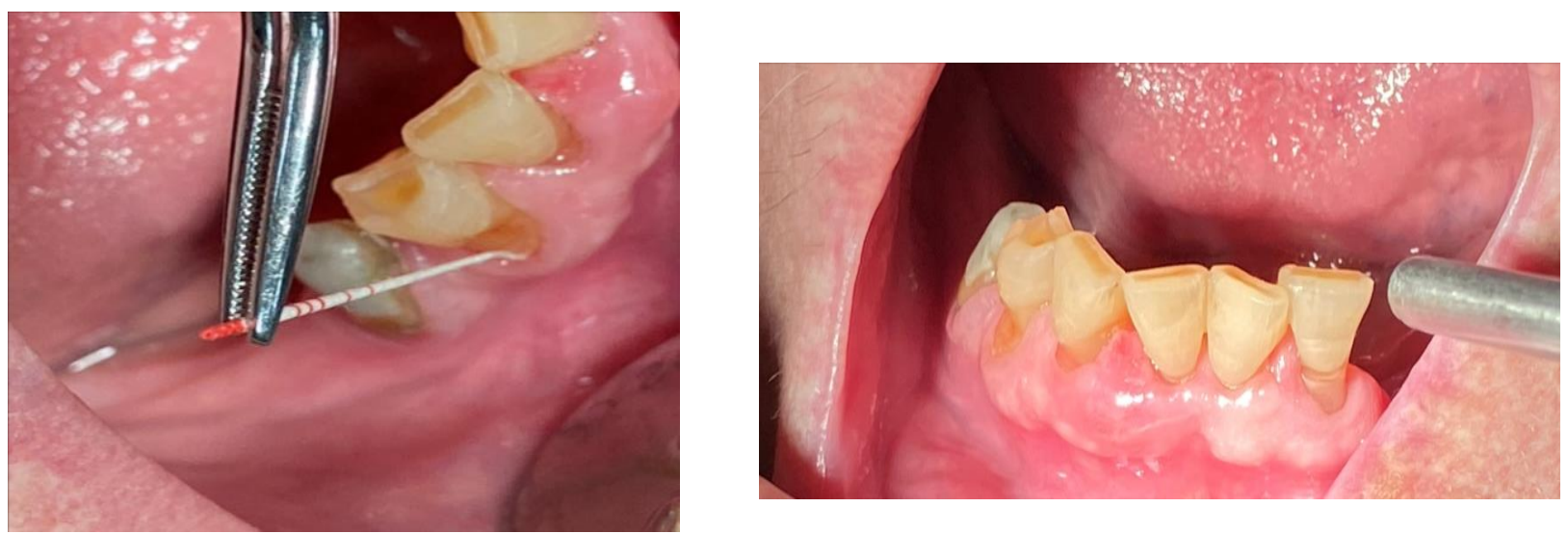

Figure 5 shows the way of recording gingival fluid values; The picture shows the way of collecting gingival fluid by placing a red paper point at the entrance of the sulcus 

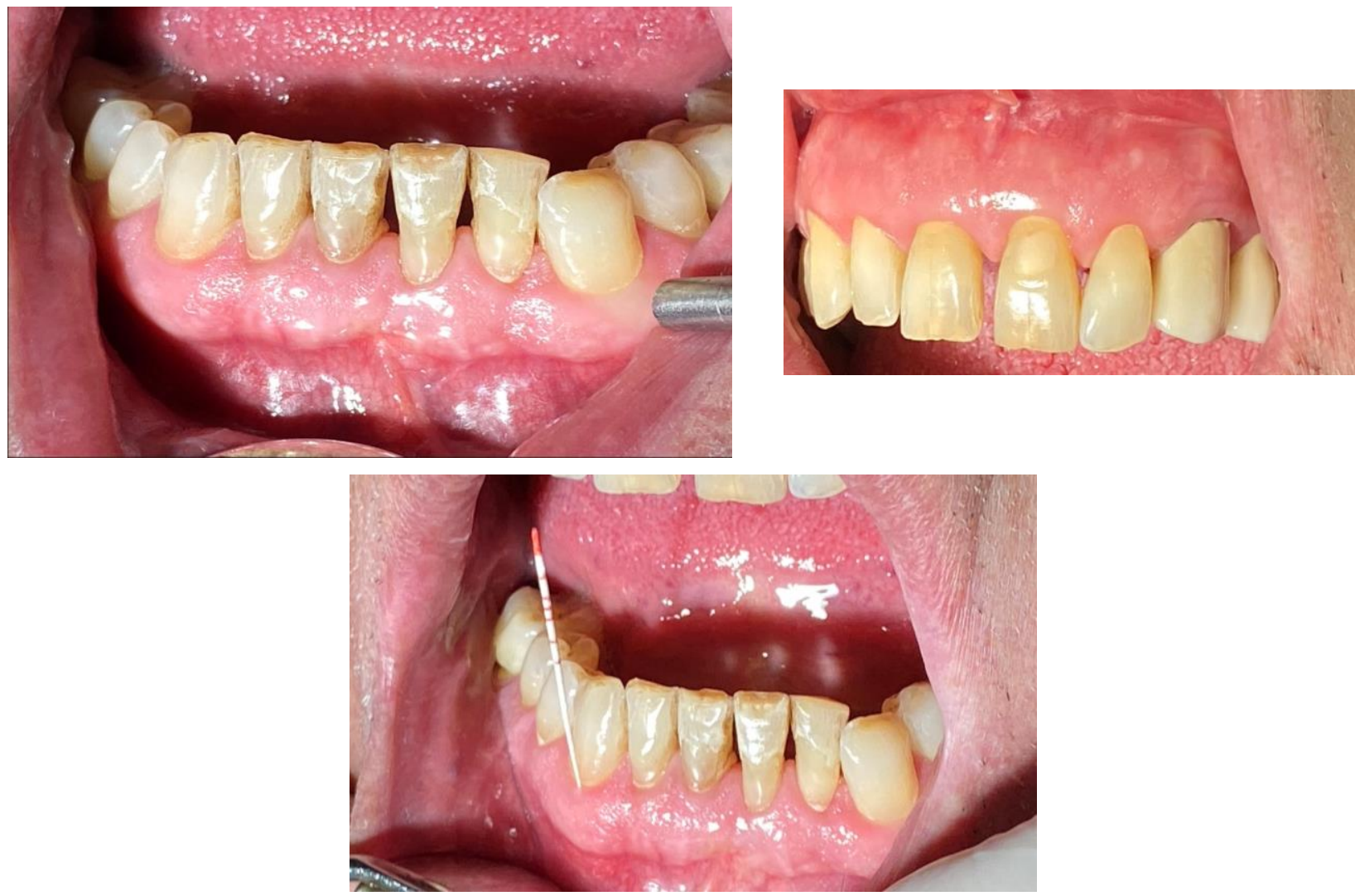

Figure 6. Patient age 53 years, with chronic periodontitis where can are distinguished the pink gingiva, gingival recession and the way of recording the amount of gingival fluid

\section{Discussions}

The results of our study collected in figures are as follows: The patient group for cross-sectional data accounted for $35 \%$ of the total patients, while the patients for retrograde data accounted for about $65 \%$ of the total patients, but these patients disaggregated by gender were randomly $50 \%$ female and 50\% gender masculine. The total number of patients of the first group and the second group divided according to age intervals, respectively were in the range of 20-30 years $56 \%$ of patients, $24 \%$ female and $30 \%$ male, the age range $31-50$ years included $24 \%$ of patients $11 \%$ male and $13 \%$ female. The age range 51 years and older were about $21 \%$ of patients with $89 \%$ male and $12 \%$ female. Expressed in cumulative percentage up to the age of 50 , the number of patients reached up to $80 \%$. The highest percentage was in males in the age range of 20-30 years with $30 \%$. Higher education includes about $57 \%$ of the total patients for the first group and for the second group, while secondary education was at $43 \%$.

The highest value for the level of higher education was in the male group retrograde group, while for the level of secondary education was in the first group of cross-sectional data in the female group. Higher education at the highest value $39 \%$ of patients reaches the age range $20-30$ years, while the lowest value at the value of $8 \%$ at the age range 51 years high. Secondary education has almost the same value of $\%$ of patients about $13-14 \%$ despite the age intervals of first and second group patients. On specific data on the oral status of patients: If we rely on the number of natural teeth in the oral cavity as a characteristic evaluative sign for the presence of chronic periodontitis, $11 \%$ of patients had the highest value in females, and $8 \%$ in males. There are various studies in the literature that support this fact; where $52 \%$ of patients are in the category with 20-31 natural teeth in the oral cavity, where $28 \%$ are female and $23 \%$ are male.

Interest in replacing missing teeth seems higher in females expressed in $22 \%$ of patients of this gender with replaced teeth, while $17 \%$ in males. Chronic periodontitis according to the index of gingival hemorrhage, was higher in females at $15 \%$ and more reduced to $3 \%$ in males in the group for cross-sectional data. In the cross-sectional data set, chronic periodontitis appears according to the gingival bleeding index, amounting to $18 \%$. In the group of patients for retrograde data, chronic periodontitis according to the index of gingival hemorrhage reaches the value of $28 \%$, $19 \%$ of females and $9 \%$ of males. The highest amount of gingival fluid is within the $0-3 \mathrm{~mm}$ range in the mandibular canine in $47 \%$ of patients.

Whereas in the maxillary molar there are two intervals $0-3 \mathrm{~mm}$ with $38 \%$ of patients and $7 \mathrm{~mm}$ - yes with $38 \%$ of patients, for patients of the first group for cross-sectional data. In the second group of patients for retrograde data, the mandibular canine includes about 53\% of patients with a range of $0-3 \mathrm{~mm}$ gingival fluid and $41 \%$ for the maxillary molar in the range of $0-3 \mathrm{~mm}$. In contrast to cross-sectional data, after the $0-3 \mathrm{~mm}$ interval to the maxillary molar is the 
4-6mm interval with $26 \%$ of patients. In the mandibular canine the amount of gingival fluid separated from the pathology for chronic periodontitis in the ratio 50\%: 50\% in pathology depending on gravity to 50\%, 50\% mild pathology and 50\% aggravated pathology. In maxillary molar the pathology rate is in the ratios 40\%: 60\% and mild pathology and aggravated pathology in the ratios 31\%: $29 \%$.

These studies even measure the fact in the assessment of the severity of the disease expressed in its severity assessed according to the relevant indices. Chronic periodontal activity is measured and evaluated quite well by looking at the results according to the hemorrhage index in the probed teeth. These studies, always according to the literature, perform this assessment according to different age groups, as it is known that the severity and severity of chronic periodontal disease depends on the age of the affected patient. Attachment loss and gingival recession are other data that record the extent of chronic periodontitis, data already recorded by Steven et al. (2009), Serrano and Suarez (2019) and Khalifehzadeh et al. (2019) [2, 13, 22]. This study was used in our study in the selection of measurements performed on the premolar in the buccal part. The number of natural teeth in the oral cavity is an indicator of oral health. They even link this indicator to the assessment of risk factors for chronic periodontitis, such as smoking $[2,4,15,17]$.

Tooth loss is characteristic of chronic periodontitis, leading to lifestyle changes, so the way the disease spreads must be known. The epidemiological goal is to apply the results obtained from the study in promoting the strengthening and improvement of health in the planning of national or regional programs of oral health promotion, to prevent and treat them. Current retrospective studies are undertaken to assess the prevalence of different forms of chronic periodontitis, data obtained from different departments of periodontology, across different states.

Depending on the localization of the chronic periodontium, localized and generalized subdivision is performed. The severity of generalized chronic periodontitis is higher and more difficult to treat. However, care must be taken in the differential diagnosis between chronic and aggressive periodontitis. The prevalence of periodontitis recorded on the basis of data from the participants is initially recorded in the e-xelit tables to then perform the analysis according to epidemiological tests. Based on studies with a relatively high number of patients where there is always a tendency for female gender balance: male. We used this fact in our study, where the total number of patients of the first group and the second group were in the ratio 50\% male and 50\% female. Among the types of periodontitis, chronic periodontitis occupies a high percentage in almost $78 \%$, including its forms, localized or generalized. The prevalence of chronic periodontitis $(90.35 \%)$ was significantly higher than that of aggressive periodontitis $(9.64 \%)$ in the sample population according to Kapoor et al. (2012), Velidandla et al. (2019), Nagao and Tanigawa (2019), Müller Campanile et al. (2019) and Khalifehzadeh et al. (2019) [3, 6, 12, 21, 22].

\section{Conclusion}

Both cross-sectional and retrograde data chronic periodontitis is a disease affecting young people, with a higher prevalence in females. This data is expressed in disease prevalence values gives results in high ratios of tooth loss for periodontal reasons. The highest care for replacement with fixed prosthetics again belongs to the female, but with a high bacterial plaque index. The high index of gingival hemorrhage with reduced bacterial plaque index are data with high percentages both for the group of patients cross-sectional data and for the group of patients with retrograde data. High prevalence of chronic periodontitis at a young age indicates a poor prognosis of this disease at older ages.

\section{Declarations}

\subsection{Author Contributions}

I.R. collected the scientific data and wrote the manuscript. S.H. revised and edited the manuscript. Literature research was conducted by S.H. G.L. and V.O. collected the scientific data. All authors read and approved the final manuscript.

\subsection{Funding}

The author(s) received no financial support for the research, authorship, and/or publication of this article.

\subsection{Acknowledgements}

Our thanks belong to our family. Henri and Hera drive us forward, and further in the field of scientific research.

\subsection{Ethical Approval}

All subjects gave their informed consent for inclusion before they participated in the study. The study was conducted in accordance with the Declaration of Helsinki, and the protocol was approved by the Albanian University. 


\subsection{Data Availability Statement}

The data presented in this study are available on request from the corresponding author. The data are not publicly available.

\subsection{Conflict of Interest}

The authors declare that they have no known competing financial interests or personal relationships that could have appeared to influence the work reported in this paper.

\section{References}

[1] Carranza - Clinical periodontology; Mosby (2009); Kapitulli "Klasifikimi i sëmundjeve periodontale".

[2] Steven 1. Bricker, Robert P. Langlais, Craig S. Miller (2009), "Diagnoza orale”; Shtëpia Botuese "UFO” Press.

[3] Kapoor, Anoop, Ranjan Malhotra, Vishakha Grover, and Deepak Grover. "Systemic Antibiotic Therapy in Periodontics." Dental Research Journal 9, no. 5 (2012): 505. doi:10.4103/1735-3327.104866.

[4] Carranza - Clinical periodontology; Mosby (2009); Kapitulli "Epidemiologjia e sëmundjeve periodontale".

[5] Wei, X., Zhao, H.-Q., Ma, C., Zhang, A.-B., Feng, H., Zhang, D., \& Liu, C. (2019). The association between chronic periodontitis and oral Helicobacter pylori: A meta-analysis. PLOS ONE, 14(12), e0225247. doi:10.1371/journal.pone.0225247.

[6] Velidandla, S., Bodduru, R., Birra, V., Jain, Y., Valluri, R., \& Ealla, K. K. R. (2019). Distribution of Periodontal Pockets among Smokers and Nonsmokers in Patients with Chronic Periodontitis: A Cross-sectional Study. Cureus. doi:10.7759/cureus.5586.

[7] Gomes - Filho, I. S., Cruz, S. S. da, Trindade, S. C., Passos - Soares, J. de S., Carvalho - Filho, P. C., Figueiredo, A. C. M. G., ... Scannapieco, F. (2019). Periodontitis and respiratory diseases: A systematic review with meta - analysis. Oral Diseases, 26(2), 439-446. doi:10.1111/odi.13228.

[8] Jia, L., Jia, J., Xie, M., Zhang, X., Li, T., Shi, L., ... Zhang, X. (2019). Clinical attachment level gain of lasers in scaling and root planing of chronic periodontitis: a network meta-analysis of randomized controlled clinical trials. Lasers in Medical Science, 35(2), 473-485. doi:10.1007/s10103-019-02875-5.

[9] Hou, R., Huo, X., Zhang, S., Xu, C., Huang, Y., \& Xu, X. (2020). Elevated levels of lead exposure and impact on the antiinflammatory ability of oral sialic acids among preschool children in e-waste areas. Science of the Total Environment, 699, 134380. doi:10.1016/j.scitotenv.2019.134380.

[10] Fine, D. H., Armitage, G. C., Genco, R. J., Griffen, A. L., \& Diehl, S. R. (2019). Unique etiologic, demographic, and pathologic characteristics of localized aggressive periodontitis support classification as a distinct subcategory of periodontitis The Journal of the American Dental Association, 150(11), 922-931. doi:10.1016/j.adaj.2019.07.024.

[11] Coelho, J. M. F., Miranda, S. S., da Cruz, S. S., Trindade, S. C., Passos-Soares, J. de S., Cerqueira, E. de M. M., ... GomesFilho, I. S. (2019). Is there association between stress and periodontitis? Clinical Oral Investigations, 24(7), 2285-2294 doi:10.1007/s00784-019-03083-9.

[12] Nagao, Y., \& Tanigawa, T. (2019). Red complex periodontal pathogens are risk factors for liver cirrhosis. Biomedical Reports. doi:10.3892/br.2019.1245.

[13] Serrano, C., \& Suarez, E. (2019). Prevalence of Severe Periodontitis in a Colombian Adult Population. Journal of the International Academy of Periodontology, 21(2), 53-62.

[14] Iordanishvili, A. K., Pikhur, O. L., Malina, M. S., \& Tityuk, S. Y. (2019). Prevalence, clinical and morphological features of tooth root caries in the adult human. Stomatologiya, 98(4), 38. doi:10.17116/stomat20199804138.

[15] Czesnikiewicz-Guzik, M., Osmenda, G., Siedlinski, M., Nosalski, R., Pelka, P., Nowakowski, D., ... Guzik, T. J. (2019). Causal association between periodontitis and hypertension: evidence from Mendelian randomization and a randomized controlled trial of non-surgical periodontal therapy. European Heart Journal, 40(42), 3459-3470. doi:10.1093/eurheartj/ehz646.

[16] Wallace, K., Shafique, S., \& Piamjariyakul, U. (2019). The Relationship between Oral Health and Hemodialysis Treatment among Adults with Chronic Kidney Disease: A Systematic Review. Nephrology Nursing Journal, 46(4), 375-394.

[17] Lee, Y. H., Lew, P. H., Cheah, C. W., Rahman, M. T., Baharuddin, N. A., \& Vaithilingam, R. D. (2019). Potential mechanisms linking periodontitis to rheumatoid arthritis. Journal of the International Academy of Periodontology, 21(3), 99110 .

[18] Hagenfeld, D., Zimmermann, H., Korb, K., El-Sayed, N., Fricke, J., Greiser, K. H., ... Becher, H. (2019). Periodontal Health and Use of Oral Health Services: A Comparison of Germans and Two Migrant Groups. International Journal of Environmental Research and Public Health, 16(16), 3000. doi:10.3390/ijerph16163000. 
[19] Akula, M., Prathypaty, S., Darapla, A., Dhulipala, M., \& Vedula, C. (2019). Prevalence of different forms of periodontitis in patients visiting Government Dental College and Hospital, Hyderabad, since last decade: A retrospective study. Journal of Indian Society of Periodontology, 23(4), 367. doi:10.4103/jisp.jisp_698_18.

[20] Santos - Paul, M. A., Neves, R. S., Gowdak, L. H. W., de Paula, F. J., David - Neto, E., Bortolotto, L. A., ㅋ De Lima, J. J. G. (2019). Cardiovascular risk reduction with periodontal treatment in patients on the waiting list for renal transplantation. Clinical Transplantation, 33(8). doi:10.1111/ctr.13658.

[21] Müller Campanile, V., Megally, A., Campanile, G., Gayet - Ageron, A., Giannopoulou, C., \& Mombelli, A. (2019). Risk factors for recurrence of periodontal disease in patients in maintenance care in a private practice. Journal of Clinical Periodontology, 46(9), 918-926. doi:10.1111/jcpe.13165.

[22] Khalifehzadeh, S., Haghanifar, S., Jenabian, N., Kazemi, S., \& Hajiahmadi, M. (2019). Clinical and radiographic evaluation of applying $1 \%$ metformin biofilm with plasma rich in growth factor (PRGF) for treatment of two-wall intrabony periodontal defects: A randomized clinical trial. Journal of Dental Research, Dental Clinics, Dental Prospects, 13(1), 51-56. doi:10.15171/joddd.2019.008. 